\title{
Computational networks of activating transcription factor 3 gene in Huh7 cell lines and hepatitis $C$ virus-infected Huh7 cell lines
}

\author{
JINGKUN LIU ${ }^{1}$, BING WANG ${ }^{2}$, WENJUN WANG ${ }^{1}$, MINGZHU SUN $^{1}$, YAPPING LI ${ }^{1}$, \\ XIAOLI JIA ${ }^{1}$, SONG ZHAI ${ }^{1}$ and SHUANGSUO DANG ${ }^{1}$ \\ ${ }^{1}$ Department of Infectious Diseases, The Second Affiliated Hospital of Medical School of Xi'an Jiaotong University, \\ Xi'an, Shaanxi 710004; ${ }^{2}$ Department of the Laboratory, Shaanxi Province Health Inspection Institution, \\ Xi'an, Shaanxi 710077, P.R. China
}

Received March 13, 2014; Accepted March 12, 2015

DOI: $10.3892 / \mathrm{mmr} .2015 .3548$

\begin{abstract}
Activating transcription factor 3 (ATF3) is an adaptive-response gene of the ATF family. ATF3 activity may be induced in response to a number of different stress-associated signals and ATF3 is involved in a variety of cellular processes. However, the functions of ATF3 and its molecular networks in human hepatoma cells lines and hepatitis $\mathrm{C}$ virus-infected Huh7 (HCV-Huh7) cells are not well understood. In the present study, ATF3 regulatory networks in Huh7 and HCV-Huh7 cell lines were established using the linear programming-based GRNinfer software and molecule annotation system 3.0 software. The gene expression omnibus dataset, GSE20948, was analyzed. The resulting network consisted of clusters located upstream and downstream of ATF3 in Huh7 and HCV-Huh7 cell lines. Using the annotation, visualization and integrated discovery (DAVID) software, 10 activation and 2 inhibition enriched functional annotation clusters were identified downstream of ATF3 in HCV-Huh7 cells. However, there were no enriched functional annotation clusters identified upstream of ATF3 in HCV-Huh7 cells. Furthermore, no clusters were identified downstream nor upstream of ATF3 in Huh7 cells. Gene ontology term and Kyoto encyclopedia of genes and genomes pathway analyses demonstrated that ATF3 may be involved in a number of biological processes, in particular, in metabolism regulation in HCV-Huh7 cells. It is hypothesized that the ATF3 pathway may be activated in Huh7 cells following $\mathrm{HCV}$ infection and that it is a potential 'hub' in the network of HCV-Huh7 cells.
\end{abstract}

Correspondence to: Mr. Shuangsuo Dang, Department of Infectious Diseases, The Second Affiliated Hospital of Medical School of Xi'an Jiaotong University, 157 Xi'wu Road, Xi'an, Shaanxi 710004, P.R. China E-mail: dang212@126.com

Key words: activating transcription factor 3, computational network, Huh7 cell line, hepatitis C virus-infected Huh7 cell line

\section{Introduction}

Hepatitis C virus (HCV) infection affects 170 million people worldwide and as a major cause of liver disease worldwide, $\mathrm{HCV}$ is a potential cause of substantial morbidity and mortality in the future and therefore, a health serious health concern (1). Approximately $80 \%$ of patients with HCV fail to clear the virus and $<10 \%$ may develop severe liver diseases, such as chronic hepatitis, liver cirrhosis and hepatocarcinoma, which reduce the chance of patient survival (2). Understanding HCV pathology and the development of novel drugs with which to treat patients with $\mathrm{HCV}$ is an ongoing challenge.

Microarrays and different types of high-throughput approaches, for example, high-throughput RNA-Seq and highthroughput DNA-Seq, for gene expression analysis have led to an improved understanding of the processes underlying $\mathrm{HCV}$ infection. These approaches are advantageous compared with traditional approaches, which are restricted to analyzing small numbers of genes (3). Microarray technology does not require gene selection in advance, meaning that the method is less biased and is capable of identifying genes that are modified when cells are exposed to environmental changes (4). Microarray technology aims to establish gene regulatory networks and to identify interactions among genes and their products. Carefully analyzed networks are used to identify correlated genes that are associated with the same biological processes or pathways, and to infer interactions among molecules, such as physical association, metabolite flow, regulatory associations and co-expression (5). In the present study, GRNinfer software was used in order to establish a gene regulatory network and to identify molecular interactions, including activation and inhibition, in the activating transcription factor 3 (AFT3) signaling pathway in healthy Huh7 (Huh7) and HCV-infected (HCV-Huh7) cell lines.

\section{Materials and methods}

Microarray. The gene expression profile data GSE 20948 (http://www.ncbi.nlm.nih.gov/geo/query/acc. cgi? $a c c=G S E 20948)$ were extracted from the public Gene Expression Omnibus database (http://www.ncbi.nlm.nih. gov/gds/). Microarray analysis was performed for 14 Huh7 
samples ( 2 samples, 6 h; 3 samples, 12 h; 3 samples, 18 h; 3 samples, 24 h; 3 samples, 48 h.) and 14 HCV-Huh7 samples (2 samples, 6 h; 3 samples, 12 h; 3 samples, 18 h; 3 samples, $24 \mathrm{~h}$; 3 samples, $48 \mathrm{~h}$ ); the latter had been infected with HCV for $6,12,18,24$ or $48 \mathrm{~h}$. The platform of the data was GPL 570 (HG-u133-plus-2) affymetrix human Genome u 1332.0 array.

Gene selection algorithms. Fifty HCV-Huh7 molecular markers were identified using Multiexperiment Viewer (http://www. tm4.org/mev.html; Version 4.8.1-windows), which belongs to TM4, in order to conduct a significant analysis of microarrays (SAM). The TM4 suite consists of four major applications, including Microarray Data Manager, TIGR_Spotfinder, Microarray Data Analysis System. This software is a free, open-source software released under the Artistic license, and is OSI certified. SAM is a statistical technique, used to identify significant genes in a set of microarray experiments. The explanatory variable is gene expression measurement from a set of microarray experiments, and the response variable is a grouping, such as mock-infected or infected. Microarray raw data CEL files were processed using expression console software of Affymetrix, Inc. (Santa Clara, CA, USA). In the present study, 50 significant genes using ATF3 as a target gene, which was on interest, were further analyzed.

Network establishment of candidate genes. ATF3 gene networks were constructed using GRNinfer (6) and GVedit (http://portableapps.com/node/38245; version: 2.38) tools. GRNinfer is a novel mathematical method base on linear programming and a decomposition procedure that infers gene networks. The method ensures derivation of the most consistent network structure reducing the issues associated with data scarcity, yet improving reliability. The following equation represents all of the possible networks for the same dataset: $J=\left(X^{\prime}-A\right) U \wedge^{1} V^{\mathrm{T}}+Y V=J+Y V^{\mathrm{T}}$. Where $J=\left(J_{i j}\right) m \mathrm{x} m=\partial f(x) / \partial x$ is an $n \times m$ Jacobian matrix or connectivity matrix, $X=\left(x\left(t_{1}\right), \ldots, x\left(t_{m}\right)\right)$ and all $n \times m$ matrices with $x_{i}^{\prime}\left(t_{j}\right)=\left[x_{i}\left(t_{j}+1\right)-x_{i}\left(t_{j}\right)\right] /\left[t_{j}+1-t_{j}\right]$ for $i=1, \ldots, n ; j=1, \ldots . m . X(t)=\left(x_{l}(t), \ldots, x n(t)^{T} \sum R^{n}, a=\left(a_{1} \ldots, a_{n}\right)^{\mathrm{T}} \sum R n, x i(t)\right.$ is the expression level (mRNA concentrations) of gene $i$ at time instance $t . y=(y i j)$ is an $n \times n$ matrix, where $y_{i j}$ is zero if $\mathrm{e}_{j}=0$. $U$ is a unitary $m \times n$ matrix of left eigenvectors, $\Lambda=\operatorname{diag}\left(e 1, \ldots, e_{\mathrm{n}}\right)$ is a diagonal $n \times n$ matrix containing the $n$ eigenvalues and $V^{\mathrm{T}}$ is the transpose of a unitary $n \times n$ matrix of right eigenvectors.

The parameters were $\lambda, 0.0$ and threshold, $1 \times 10^{-9}$.

Functional annotation clustering. The database for annotation, visualization and integrated discovery (DAVID; http://www.david.niaid.nih.gov) was used. The DAVID gene function clustering tool provides representative annotation and gene ontology (GO) term enrichment analysis, which separates genes into different enrichment score groups according to the gene annotation collective frequency occurrence $(7,8)$.

Molecule annotation system 3.0 (MAS 3.0). MAS (http://bioinfo. capitalbio.com/mas3/) is an analysis platform, which adds biological function annotation to high throughput microarray data. By integrating the relevant annotation information from a number of public information databases, MAS synthesizes biological data, including genes, proteins, functions, expression, protein interactions, signaling pathways, diseases and methylation.
MAS enables the incorporation of genetic data from Genebank, European molecular biology laboratory, SwissPort, GO, KEGG, BioCarta, gene map annotator and pathway profiler (GenMapp), mirBase, expected progeny differences, HRPD, MIND, BIND, Intact, TRANSAC, UniGene, single nucleotide polymorphism database, OMIM, InterPro, HUGO, mouse genome informatics and rat genome database.

\section{Results}

Gene enrichment analyses. ATF3 was one of fifty genes which were significantly differentially expressed between Huh7 and HCV-Huh7 cell lines (fold change=8.782885; Table I). Gene GO term enrichment analysis for ATF3 demonstrated that the molecular function of ATF3 is associated with transcription corepressor activity, protein binding, sequence-specific DNA binding and protein dimerization activity. ATF3 biological processes are associated with DNA-dependent transcription regulation. ATF3 cellular component is localized in the nucleus and nucleolus. GenMAPP analysis demonstrated that ATF3 is associated with smooth contraction, hypertrophy model, NetPath 5 and Hs transforming growth factor $\beta 1$ (TGF- $\beta$ ) NetPath 7, and that it exhibits transcription cofactor activity. Disease analysis demonstrated that ATF3 is associated with shock, leukemia, colorectal cancer, neoplasm metastasis, nervous system disease, stomach cancer, necrosis, leukemia T cell, lymphoma non-hodgkins, malignant neoplasm of the breast, hyperalgesia, episodic ataxia type 2 and hereditary ataxia overview.

Identification of genes upstream and downstream of ATF3. Using GRNInfer, a cell network was constructed, which included gene clusters upstream and downstream of ATF3 in Huh7 and HCV-Huh7 cell lines. According to DAVID software analysis, 12 gene clusters were identified downstream of ATF3 in HCV-Huh7 cells (10 activation and 2 inhibition; Table II). Using an MAS 3.0 software GO analysis, ATF3 annotation regulation networks were enriched. The upstream pathway of ATF3 in Huh7 cells included the activation of stanniocalcin 2 cationic amino acid transporter, Y+ system member 1 (SLC7A1) and insulin receptor substrate 2 (IRS2), involving 19 GO terms and three KEGG pathways, and inhibition of four and a half LIM domains 2 (FHL2), involving five GO terms. The downstream pathway of ATF3 in Huh7 cells included inhibition of zinc finger protein 295 (ZNF295), involving two GO terms. The upstream pathway of ATF3 in HCV-Huh7 cells included the activation of inhibin $\beta \mathrm{E}$ chain (INHBE), asparagine synthetase (ASNS) and SLC7A1, involving ten GO terms and four KEGG pathways, and the inhibition of reticulocalbin 1, EF-hand calcium binding domain (RCN1), involving one GO term. The downstream pathway of in HCV-Huh7 cells included the activation of the following genes: Brain-derived neurotrophic factor, cold inducible RNA binding protein, FHL2, fatty acid binding protein 3, interferon regulatory factor 9, InaD-like (INADL), IRS2, IRS1, kruppel-like factor 10, LOC100506392, LY6/PLAUR domain containin 1, prion protein, peroxisome proliferator-activated receptor $\gamma$ coactivator 1- $\alpha$, RAR-related orphan receptor A, phospholipase A1 member A, SFT2 domain containing 3, SMAD family member 5 , TGF $\beta 1$ induced transcript 1 , 
Table I. Fifty genes exhibiting significant differential expression between Huh7 and HCV-Huh7 cell lines identified using SAM.

\begin{tabular}{|c|c|c|}
\hline Gene ID & Gene & Fold change \\
\hline 201010_s_at & TXNIP & 21.518297 \\
\hline 203438_at & STC2 & 20.873947 \\
\hline 210587_at & Inhibin $\beta \mathrm{E}$ chain & 19.26132 \\
\hline 201008_s_at & TXNIP & 15.231962 \\
\hline 203439_s_at & STC2 & 14.20261 \\
\hline 201009_s_at & TXNIP & 13.7505045 \\
\hline 238029_s_at & SLC 16, member 14 & 11.429825 \\
\hline 205047_s_at & Asparagine synthetase & 11.315236 \\
\hline 231202_at & Aldehyde dehydrogenase family 1 member & 11.254294 \\
\hline 225283_at & Arrestin domain-containing protein 4 & 10.549104 \\
\hline 228653_at & SAMD5 & 9.061874 \\
\hline 202672_s_at & Activating transcription factor 3 & 8.782885 \\
\hline 201300_s_at & Prion protein & 7.5039783 \\
\hline 212909_at & LY6/PLAUR domain containin 1 & 7.415823 \\
\hline 218332_at & Brain expressed, X-linked 1 & 7.3808937 \\
\hline 203372_s_at & SOCS2 & 7.347132 \\
\hline 219195_at & Peroxisome proliferator-activated receptor $\gamma$ coactivator $1-\alpha$ & 6.9846096 \\
\hline 212810_s_at & SLC 1 member 4 & 6.877748 \\
\hline 210426_x_at & RORA & 6.6006436 \\
\hline 209183_s_at & Chromosome 10 open reading frame 10 & 6.530727 \\
\hline 226682_at & RORA & 6.4361844 \\
\hline 202393_s_at & Kruppel-like factor 10 & 6.4241524 \\
\hline 214285_at & Fatty acid binding protein 3 & 6.3771186 \\
\hline 203882_at & Interferon regulatory factor 9 & 6.129926 \\
\hline 221523_s_at & Ras-related glutamic-pyruvate transaminase binding D & 6.011061 \\
\hline 212295_s_at & SLC 7 (cationic amino acid transporter, Y+ system) member 1 & 5.976633 \\
\hline 1554008_at & Oncostatin M receptor & 5.8743997 \\
\hline 218851_s_at & SFT2 domain containing 3, WD repeat domain 33 & 5.831587 \\
\hline 223681_s_at & InaD-like & 5.778195 \\
\hline 203373_at & SOCS2 & 5.741121 \\
\hline 209651_at & Transforming growth factor $\beta 1$ induced transcript 1 & 5.6655536 \\
\hline 201063_at & Reticulocalbin 1, EF-hand calcium binding domain & 5.624495 \\
\hline 1569433_at & SAMD5 & 5.4978223 \\
\hline 206382_s_at & Brain-derived neurotrophic factor & 5.459223 \\
\hline 221530_s_at & Basic helix-loop-helix family, member e41 & 5.424661 \\
\hline 228708_at & Ras-related protein Rab-27B & 5.3274593 \\
\hline 209185_s_at & IRS 2 & 5.273203 \\
\hline 209610_s_at & SLC 1 member 4 & 5.230212 \\
\hline 214755_at & UDP-N-acteylglucosamine pyrophosphorylase 1-like 1 & 5.206161 \\
\hline 202847_at & Phosphoenolpyruvate carboxykinase 2 & 5.199079 \\
\hline 227037_at & Phospholipase D family, member 6 & 5.078215 \\
\hline 225539_at & Zinc finger protein 295 & 5.0584846 \\
\hline 210479_s_at & RORA & 5.0370793 \\
\hline 212290_at & SLC 7 (cationic amino acid transporter, Y+ system) member 1 & 5.0187483 \\
\hline 228519_x_at & Cold inducible RNA binding protein & 4.95052 \\
\hline 219584_at & Phospholipase A1 member A & 4.935018 \\
\hline 242979_at & IRS 1 & 4.919549 \\
\hline 202949_s_at & Four and a half LIM domains 2 & 4.856401 \\
\hline 1568813_at & LOC100506392 & 4.792747 \\
\hline 212811_x_at & SLC 1 member 4 & 4.7892227 \\
\hline
\end{tabular}

TXNIP, thioredoxin interacting protein; STC2, stanniocalcin 2; SAMD5, sterile $\alpha$ motif domain containing 5; SOCS2, suppressor of cytokine signaling 2; SLC, solute carrier family; RORA, RAR-related orphan receptor A; IRS, insulin receptor substrate; SAM, significant microarrays; HCV, hepatitis virus; Huh7 cell line, hepatoma cell line; HCV-Huh7 cell line, HCV-infected Huh7 cell line. 


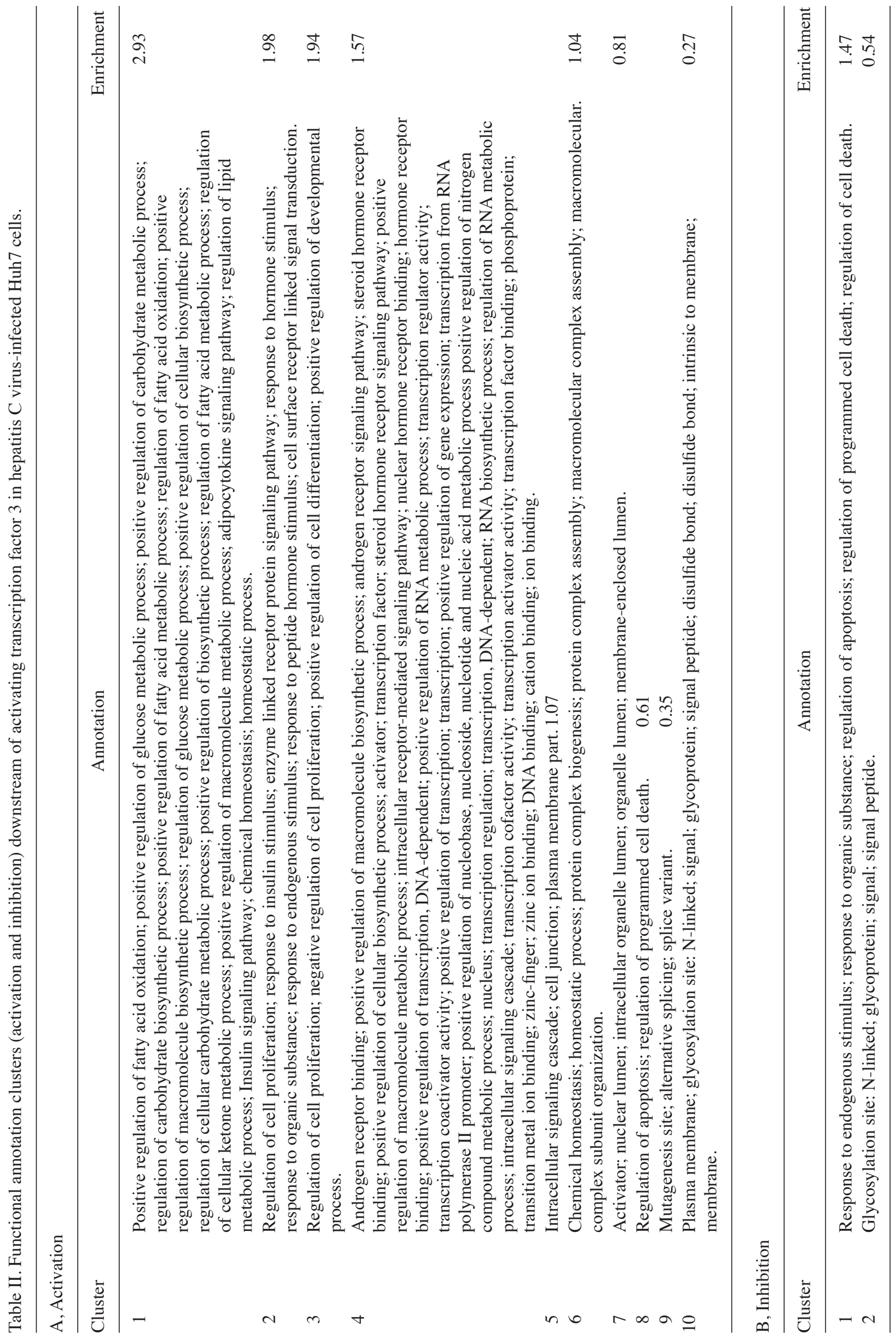




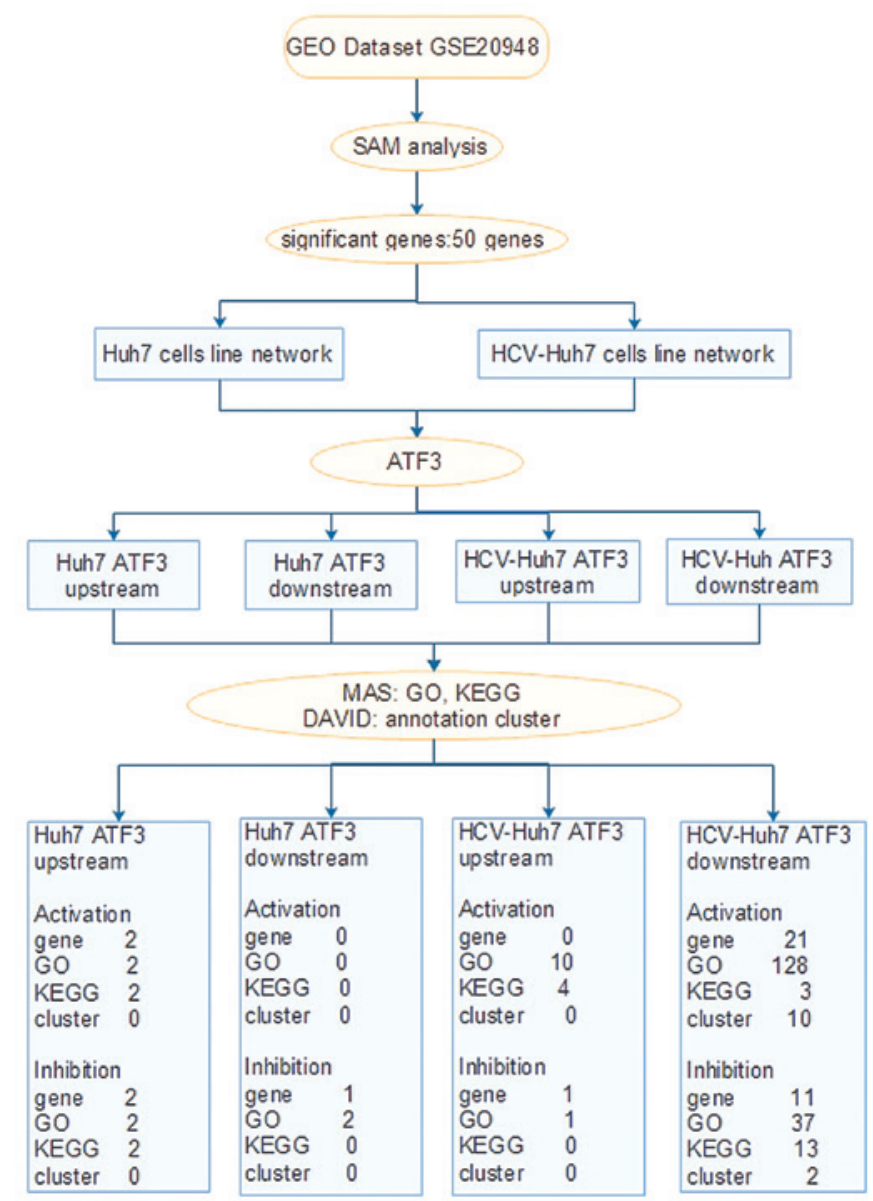

Figure 1 ATF3 network construction and analysis processes. ATF3, activating transcription factor 3; GO, gene ontology; TXNIP, thioredoxin interacting protein; STC2, stanniocalcin 2; SAMD5, sterile $\alpha$ motif domain containing 5 , SOCS2, suppressor of cytokine signaling 2; SLC, solute carrier family; RORA, RAR-related orphan receptor A; IRS, insulin receptor substrate; DAVID, database for annotation, visualization, and integrated discovery; KEGG, kyoto encyclopedia of genes and genomes; SAM, significant analysis of microarrays; GEO, gene expression omnibus; $\mathrm{HCV}$, hepatitis $\mathrm{C}$ virus; $\mathrm{HCV}$-Huh7 cells, HCV-infected Huh7 cells; Huh7 cells, hepatoma cells.

UDP-N-acteylglucosamine pyrophosphorylase 1-like 1, ZNF295 and SLC7A1, involving 128 GO terms and 13 KEGG pathways. The downstream pathway in HCV-Huh7 cells included the inhibition of the following genes: SLC7A1, arrestin domain-containing protein 4 , ASNS, chromosome 10 open reading frame 10, INHBE, phosphoenolpyruvate carboxykinase 2, PCK2, RCN1, stanniocalcin 2, suppressor of cytokine signaling 2, SLC1A4 and thioredoxin interacting protein, involving $37 \mathrm{GO}$ terms and $13 \mathrm{KEGG}$ pathways.

Functional annotation clustering analyses. Ten activation and two inhibition annotation clusters were enriched downstream of ATF3, in HCV-Huh7 cells. The enrichment scores of the activation clusters ranged from 0.27-2.93, and the two inhibition clusters were 0.54 and 1.47. Among the activation clusters, the highest enrichment score (cluster 1; 2.93) was associated with positive metabolism regulation, such as fatty acid oxidation, glucose metabolism, carbohydrate metabolism, cellular carbohydrate metabolism, fatty acid metabolism, macromolecule biosynthesis, cellular
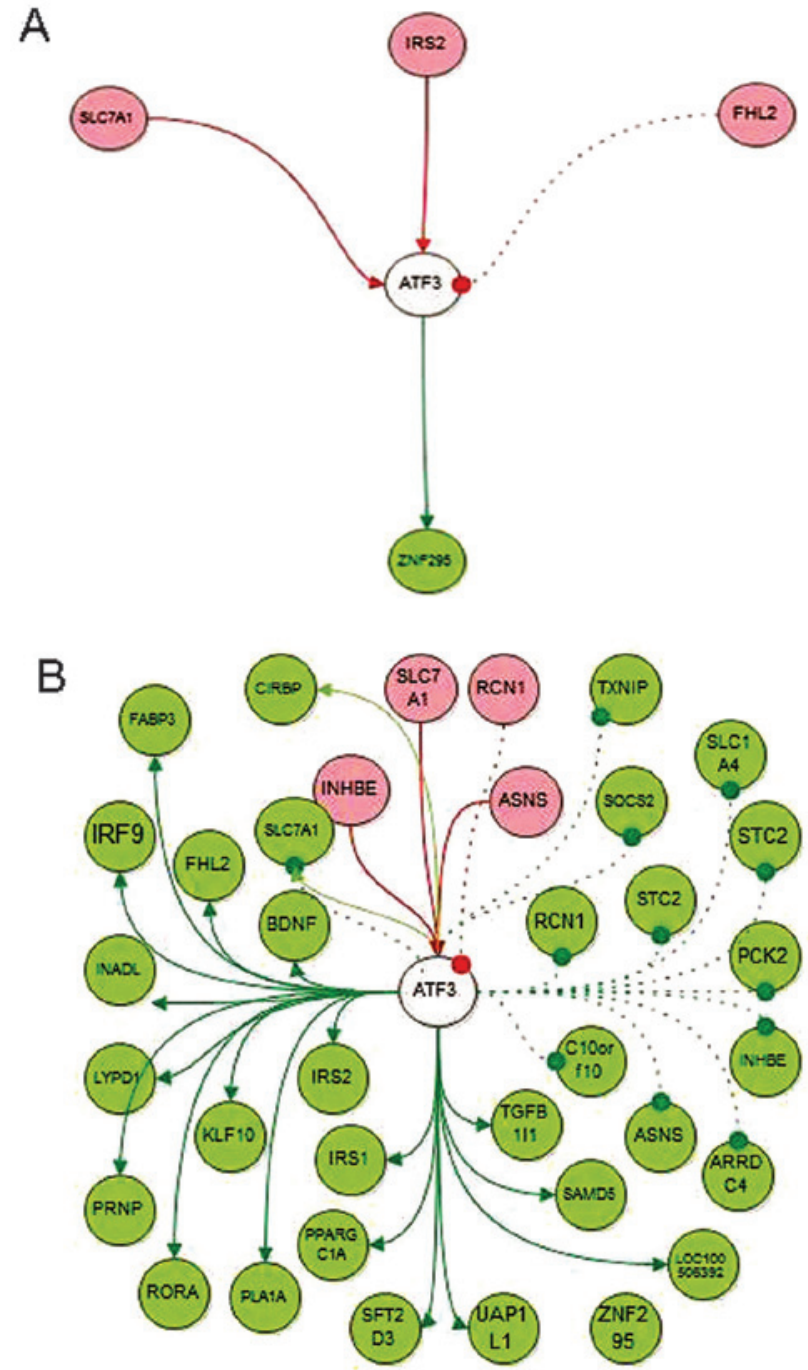

Figure 2. ATF3 network in healthy HUH7 and HCV-infected Huh7 cell lines. (A) ATF3 network in Huh7 cells; (B) ATF3 network in HCV-Huh7 cell lines. Red circle with gene name indicates the genes upstream of ATF3; Green circle with gene name indicates the genes downstream of ATF3; Red solid line with red arrow indicates the activation of ATF3 by the gene upstream to ATF3; Red dashed line with red dot indicates the inactivation of ATF3 by the gene upstream; Green solid line with green arrow indicates the activation role of the gene in the downstream by ATF3; Green dashed line with green dot indicate the inhibition of the gene in the downstream by ATF3. ATF3, activating transcription factor $3 ; \mathrm{HCV}$, hepatitis $\mathrm{C}$ virus; TXNIP, thioredoxin interacting protein; STC2, stanniocalcin 2; SAMD5, sterile $\alpha$ motif domain containing 5; SOCS2, suppressor of cytokine signaling 2; SLC1A4, solute carrier family member 4; RORA, RAR-related orphan receptor A; IRS, insulin receptor substrate. INHBE, inhibin $\beta \mathrm{E}$; ASND, asparagine synthetase; ARRDC4, arrestin domain-containing protein 4; LYPD1, LY6/PLAUR domain containin 1; PPARGC1A, peroxisome proliferator-activated receptor $\gamma$ coactivator 1- $\alpha$; KLF10, kruppel-like factor 10; FABP3, fatty acid binding protein 3; IRF9, interferon regulatory factor 9; SLC7A1, SLC 7 (cationic amino acid transporter, Y+ system) member 1; C10orf10, chromosome 10 open reading frame 10; SFT2D3, SFT2 domain containing 3 , INADL, inaD-like; TGTB1I1, transforming growth factor $\beta 1$ induced transcript 1; RCN1, reticulocalbin 1, EF-hand calcium binding domain; BDNF, brain-derived neurotrophic factor; UAP1L1, UDP-N-acteylglucosamine pyrophosphorylase 1-like 1; PCK2, phosphoenolpyruvate carboxykinase 2; ZNF295, zinc finger protein 295; CIRBP, cold inducible RNA binding protein; PLA1A, phospholipase A1 member A; FHL2, four and a half LIM domains 2; PRNP, prion protein.

biosynthesis, lipid metabolism, fatty acid metabolic regulation, macromolecule metabolism and androgen receptor 
binding. The activation clusters exhibiting enrichment scores 0.27-1.98 were associated with regulating cell proliferation, transcription, DNA-dependent developmental processes, gene expression, responses to stimuli of insulin, endogenous organic substances, peptide hormones, androgen receptor binding, androgen receptor signaling pathways, steroid hormone receptor binding, nuclear hormone receptor binding, intracellular signaling cascades, zinc-finger transcription factors and metal-binding. Therefore, it is hypothesized that ATF3 is inactive in Huh7 cells and is activated upon $\mathrm{HCV}$ infection, thereby regulating a number of cytokine functions, metabolic pathways and signal transduction. The present study identified 4 genes that were upstream of ATF3 and 33 that were downstream of ATF3 in HCV-Huh7 cells. Therefore, ATF3 may be an important regulatory factor involved in the pathological reactions of Huh7 cells undergoing the initial stages of infection with HCV.

Fig. 1 demonstrates the ATF3 network construction and analysis processes.

\section{Discussion}

In order to understand the involvement of ATF3 in HCV-infected Huh7 cells, an ATF3 GO network was established for Huh7 and $\mathrm{HCV}-\mathrm{Huh} 7$ cell lines. HCV infection leads to complex biological responses and, therefore, the subsequent genetic interactions in the infected cells are typically non-linear. However, gene networks may only be predicted based on linear equations, due to the limited understanding of biological processes required for more complex statistical analyses (9-12). In the present study, GRNinfer, a linear programming and decomposition software, was used in order to analyze and compare ATF3 networks in Huh7 and HCV-Huh7 cell lines. Constructed networks included upstream and downstream gene clusters of ATF3 in Huh7 and HCV-Huh7 cell lines (Fig. 2). Furthermore, using DAVID and MAS 3.0 software, the KEGG pathways and GO terms of four gene clusters were enriched. The results of the present study demonstrated that downstream pathways of ATF3 in HCV-Huh7 cells were significantly enhanced.

The results of the present study suggested that ATF3 may be inactive in Huh7 cells and activated following HCV infection of Huh7 cells. These results are in accordance with those observed in previous studies. ATF3 expression levels were found to be low in other cell lines or tissue (13) and were shown to rise when cells were subjected to a number of different stresses, including ischemia and trauma, as well as exposure to toxic chemicals. ATF3 mRNA expression was not detected, or remained at low levels, in a number of cell types (14). The present study demonstrated that 4 genes were upstream or downstream of ATF3 in Huh7 cells, whereas 36 genes were upstream or downstream of ATF3 in HCV-Huh7 cells. Therefore, ATF3 may be inactive in healthy Huh7 cells while it is activated in HCV-infected Huh7 cells (Fig. 2). These observations require further investigation.

Four genes were identified upstream, while 33 genes were downstream of ATF3 in HCV-Huh7 cells. Therefore, ATF3 may be associated with HCV pathology in Huh7 cells. A previous study suggested that ATF3 may represent an immediate early responsive gene, which is predominantly activated at a transcriptional level (15).
Extensive studies have shown that ATF3 is involved in immune regulation (16-23), endocrine regulation (24-27), tumorigenicity (28-32), apoptosis, cell cycling (33-37) and inflammation $(17,34,38-43)$. This suggests that ATF3 is associated with host defensive responses to inflammation, viruses and cancer. These processes require the interactions of certain cellular pathways, which influence disease outcomes. ATF3 is an adaptive-response gene, which is involved in a number of cellular processes, and accommodates cellular changes by transducing signals from various receptors via activating or repression of gene expression. ATF3 may also regulate host defense mechanisms (13). According to DAVID functional annotation clustering analysis, no annotation clusters were identified downstream of ATF3 in Huh7 cells nor were there clusters identified upstream in Huh7 and HCV-Huh7 cells. However, ten activation annotation clusters were enriched downstream of ATF3 in HCV-Huh7 cells that exhibited enrichment scores from 0.27-2.93 and two inhibition annotation clusters were enriched downstream of ATF3 in HCV-Huh7 cells that exhibited enrichment scores of 0.54 and 1.47. In addition, analyses using MAS 3.0 GO and KEGG, suggested that 128 GO terms and 13 KEGG pathways are involved downstream of ATF3 in HCV-Huh7 cells and 37 GO terms and 13 KEGG pathways were inhibited. It is hypothesized that ATF3, as an adaptive-response gene, may be involved in the pathophysiological responses of Huh7 cells to HCV infection.

In addition to liver injury and hepatocellular carcinoma, $\mathrm{HCV}$ infection is associated with fatty degeneration of the liver, suggesting that hepatitis $C$ is a metabolic disease $(44,45)$. Considerable evidence suggests that $\mathrm{HCV}$ infection is associated with metabolic syndromes, which referred to constellation of problems, including insulin resistance, obesity, hypertension and hyperlipaemia. Fatty degeneration of the liver and insulin resistance may be important in $\mathrm{HCV}$-induced metabolic syndrome and a molecular mechanism underlying this process is a virus-induced metabolic disorder of fat in the liver (46). The liver is important for the control of lipogenesis, gluconeogenesis and cholesterol metabolism. A number of investigations into pathological examination have highlighted the importance of metabolic function in liver diseases. The process of lipid metabolism is important in the proliferation of $\mathrm{HCV}$ infection (47).

As a cyclic AMP response element-binding protein family member, ATF3 participates in a range of cellular processes. Recent studies have shown that changes in ATF3 activity is associated with energy balance. Homeostasis imbalance may lead to organ growth failure, retardation and metabolic disorder. A loss of ATF3 in Drosophila was found to result in chronic inflammation and primary starvation responses, including lipid overloading in the fat bodies of the larval epithelium, which may lead to energy imbalance and death (48). Kim et al (49) showed that ATF3 was involved in a cholesterol-sensing network.

In the present study, DAVID cluster analysis found that the cluster with the highest enrichment score (2.93) was downstream of ATF3 in HCV-Huh7 cells (Table II). This suggests that ATF3 not only activates lipid metabolism, but also positively regulates fatty acid metabolic, macromolecular biosynthetic and cellular biosynthetic processes. The results of the present study showed that ATF3 may influence metabolic processes in HCV-Huh7 cells. The current study provides a 
novel perspective for exploring the association of ATF3 in $\mathrm{HCV}$ infection of Huh7 cells. However, further research is required in order to explore how ATF3 is associated with metabolic processes and the regulatory functions of ATF3.

In conclusion, an ATF3 network was constructed for Huh7 and HCV-Huh7 cell lines and gene functional annotation and enrichment cluster analyses were performed. The results of the present study suggested that ATF3 is inactive in healthy Huh7 cells and activated following HCV infection. ATF3 may contribute to the initial pathological responses to $\mathrm{HCV}$ infection in Huh7 cells. ATF3 may be associated with a number of processes, in particular lipid metabolism, during acute $\mathrm{HCV}$ infection of Huh7 cells.

\section{Acknowledgements}

This study was supported by the National Natural Science Foundation in China (grant no. 81170393/H0316).

\section{References}

1. Shepard CW, Finelli L and Alter MJ: Global epidemiology of hepatitis $C$ virus infection. Lancet Infect Dis 5: 558-567, 2005.

2. Seeff LB, Hollinger FB, Alter HJ, Wright EC, Cain CM, Buskell ZJ, Ishak KG, Iber FL, Toro D, Samanta A, et al: Long-term mortality and morbidity of transfusion-associated non-A, non-B, and type C hepatitis: A National Heart, Lung, and Blood Institute collaborative study. Hepatology 33: 455-463, 2001.

3. Guo QM: DNA microarray and cancer. Curr Opin Oncol 15: 36-43, 2003.

4. Ding $C$ and Cantor CR: Quantitative analysis of nucleic acids - the last few years of progress. J Biochem Mol Biol 37: 1-10, 2004.

5. Xiong J, Lu Y, Feng J, Yuan D, Tian M, Chang Y, Fu C, Wang G, Zeng $\mathrm{H}$ and Miao $\mathrm{W}$ : Tetrahymena functional genomics database (TetraFGD): An integrated resource for Tetrahymena functional genomics. Database (Oxford) 2013: bat008, 2013.

6. Wang Y, Joshi T, Zhang XS, Xu D and Chen L: Inferring gene regulatory networks from multiple microarray datasets. Bioinformatics 22 2413-2420, 2006.

7. Huang DW, Sherman BT, Tan Q, Collins JR, Alvord WG, Roayaei J, Stephens R, Baseler MW, Lane HC and Lempicki RA: The DAVID Gene Functional Classification Tool: A novel biological module-centric algorithm to functionally analyze large gene lists. Genome Biol 8: R183, 2007.

8. Dennis G Jr, Sherman BT, Hosack DA, Yang J, Gao W, Lane HC and Lempicki RA: DAVID: Database for Annotation, Visualization, and Integrated Discovery. Genome Biol 4: 3, 2003.

9. Haeseleer F, Sokal I, Li N, Pettenati M, Rao N, Bronson D, Wechter R, Baehr W and Palczewski K: Molecular characterization of a third member of the guanylyl cyclase-activating protein subfamily. J Biol Chem 274: 6526-6535, 1999.

10. Gustafsson M, Hornquist $M$ and Lombardi A: Constructing and analyzing a large-scale gene-to-gene regulatory network - lasso-constrained inference and biological validation. IEEE/ACM Trans Comput Biol Bioinform 2: 254-261, 2005.

11. Wang L, Huang J, Jiang M and Sun L: MYBPC1 computational phosphoprotein network construction and analysis between frontal cortex of HIV encephalitis (HIVE) and HIVE-control patients. Cell Mol Neurobiol 31: 233-241, 2011.

12. Wang L, Sun L, Huang J and Jiang M: Cyclin-dependent kinase inhibitor 3 (CDKN3) novel cell cycle computational network between human non-malignancy associated hepatitis/cirrhosis and hepatocellular carcinoma (HCC) transformation. Cell Prolif 44 291-299, 2011

13. Thompson MR, Xu D and Williams BR: ATF3 transcription factor and its emerging roles in immunity and cancer. J Mol Med (Berl) 87: 1053-1060, 2009.

14. Hai T, Wolfgang CD, Marsee DK, Allen AE and Sivaprasad U: ATF3 and stress responses. Gene Expr 7: 321-335, 1999.

15. Cai Y, Zhang C, Nawa T, Aso T, Tanaka M, Oshiro S, Ichijo H and Kitajima S: Homocysteine-responsive ATF3 gene expression in human vascular endothelial cells: Activation of c-Jun NH(2)-terminal kinase and promoter response element. Blood 96: 2140-2148, 2000.
16. Kawai T and Akira S: TLR signaling. Cell Death Differ 13: 816-825, 2006.

17. Gilchrist M, Henderson WR Jr, Clark AE, Simmons RM, Ye X, Smith KD and Aderem A: Activating transcription factor 3 is a negative regulator of allergic pulmonary inflammation. J Exp Med 205: 2349-2357, 2008.

18. Swann JB, Vesely MD, Silva A, Sharkey J, Akira S, Schreiber RD and Smyth MJ: Demonstration of inflammation-induced cancer and cancer immunoediting during primary tumorigenesis. Proc Natl Acad Sci USA 105: 652-656, 2008.

19. Rakoff-Nahoum S and Medzhitov R: Regulation of spontaneous intestinal tumorigenesis through the adaptor protein MyD88. Science 317: 124-127, 2007.

20. Leitner WW, Hwang LN, deVeer MJ, Zhou A, Silverman RH, Williams BR, Dubensky TW, Ying $\mathrm{H}$ and Restifo NP: Alphavirus-based DNA vaccine breaks immunological tolerance by activating innate antiviral pathways. Nat Med 9: 33-39, 2003.

21. Scheule RK: The role of CpG motifs in immunostimulation and gene therapy. Adv Drug Deliv Rev 44: 119-134, 2000.

22. Whitmore MM, DeVeer MJ, Edling A, Oates RK, Simons B, Lindner D and Williams BR: Synergistic activation of innate immunity by double-stranded RNA and CpG DNA promotes enhanced antitumor activity. Cancer Res 64: 5850-5860, 2004.

23. Litvak V, Ramsey SA, Rust AG, Zak DE, Kennedy KA, Lampano AE, Nykter M, Shmulevich I and Aderem A: Function of C/EBPdelta in a regulatory circuit that discriminates between transient and persistent TLR4-induced signals. Nat Immunol 10: 437-443, 2009

24. Xie J and Roberson MS: 3', 5'-cyclic adenosine 5'-monophosphate response element-dependent transcriptional regulation of the secretogranin II gene promoter depends on gonadotropin-releasing hormone-induced mitogen-activated protein kinase activation and the transactivator activating transcription factor 3. Endocrinology 149: 783-792, 2008.

25. Salisbury TB, Binder AK, Grammer JC and Nilson JH: GnRH-regulated expression of Jun and JUN target genes in gonadotropes requires a functional interaction between TCF/LEF family members and beta-catenin. Mol Endocrinol 23: 402-411, 2009.

26. Mayer SI, Dexheimer V, Nishida E, Kitajima S and Thiel G: Expression of the transcriptional repressor ATF3 in gonadotrophs is regulated by Egr-1, CREB, and ATF2 after gonadotropin-releasing hormone receptor stimulation. Endocrinology 149: 6311-6325, 2008.

27. Pelzer AE, Bektic J, Haag P, Berger AP, Pycha A, Schäfer G, Rogatsch H, Horninger W, Bartsch G and Klocker H: The expression of transcription factor activating transcription factor 3 in the human prostate and its regulation by androgen in prostate cancer. J Urol 175: 1517-1522, 2006.

28. Wu X, Nguyen BC, Dziunycz P, Chang S, Brooks Y, Lefort K, Hofbauer GF and Dotto GP: Opposing roles for calcineurin and ATF3 in squamous skin cancer. Nature 465: 368-372, 2010.

29. Yin X, Dewille JW and Hai T: A potential dichotomous role of ATF3, an adaptive-response gene, in cancer development. Oncogene 27: 2118-2127, 2008.

30. Kawai M, Jin M, Nishimura J, Dewa Y, Saegusa Y, Matsumoto S, Taniai E, Shibutani M and Mitsumori K: Hepatocarcinogenic susceptibility of fenofibrate and its possible mechanism of carcinogenicity in a two-stage hepatocarcinogenesis model of rasH2 mice. Toxicol Pathol 36: 950-957, 2008.

31. Xie JJ, Xu LY, Xie YM, Zhang HH, Cai WJ, Zhou F, Shen ZY and Li EM: Roles of ezrin in the growth and invasiveness of esophageal squamous carcinoma cells. Int J Cancer 124: 2549-2558, 2009.

32. Hamdi M, Popeijus HE, Carlotti F, Janssen JM, van der Burgt C, Cornelissen-Steijger P, van de Water B, Hoeben RC, Matsuo K and van Dam H: ATF3 and Fral have opposite functions in JNKand ERK-dependent DNA damage responses. DNA Repair (Amst) 7: 487-496, 2008.

33. Huang X, Li X and Guo B: KLF6 induces apoptosis in prostate cancer cells through up-regulation of ATF3. J Biol Chem 283: 29795-29801, 2008.

34. Rosenberger CM, Clark AE, Treuting PM, Johnson CD and Aderem A: ATF3 regulates MCMV infection in mice by modulating IFN-gamma expression in natural killer cells. Proc Natl Acad Sci USA 105: 2544-2549, 2008.

35. Wang H, Mo P, Ren S and Yan C: Activating transcription factor 3 activates p53 by preventing E6-associated protein from binding to E6. J Biol Chem 285: 13201-13210, 2010. 
36. Tian Z, An N, Zhou B, Xiao P, Kohane IS and Wu E: Cytotoxic diarylheptanoid induces cell cycle arrest and apoptosis via increasing ATF3 and stabilizing p53 in SH-SY5Y cells. Cancer Chemother Pharmacol 63: 1131-1139, 2009.

37. Kashiwakura Y, Ochiai K, Watanabe M, Abarzua F, Sakaguchi M, Takaoka M, Tanimoto R, Nasu Y, Huh NH and Kumon H: Down-regulation of inhibition of differentiation-1 via activation of activating transcription factor 3 and Smad regulates REIC/Dickkopf-3-induced apoptosis. Cancer Res 68: 8333-8341, 2008.

38. Gilchrist M, Thorsson V, Li B, Rust AG, Korb M, Roach JC, Kennedy K, Hai T, Bolouri H and Aderem A: Systems biology approaches identify ATF3 as a negative regulator of Toll-like receptor 4. Nature 441: 173-178, 2006.

39. Whitmore MM, Iparraguirre A, Kubelka L, Weninger W, Hai T and Williams BR: Negative regulation of TLR-signaling pathways by activating transcription factor-3. J Immunol 179: 3622-3630, 2007.

40. Gilchrist M, Henderson WR Jr, Morotti A, Johnson CD, Nachman A, Schmitz F, Smith KD and Aderem A: A key role for ATF3 in regulating mast cell survival and mediator release. Blood 115: 4734-4741, 2010.

41. Shukla A, MacPherson MB, Hillegass J, Ramos-Nino ME, Alexeeva V, Vacek PM, Bond JP, Pass HI, Steele C and Mossman BT: Alterations in gene expression in human mesothelial cells correlate with mineral pathogenicity. Am J Respir Cell Mol Biol 41: 114-123, 2009.
42. Khuu CH, Barrozo RM, Hai T and Weinstein SL: Activating transcription factor 3 (ATF3) represses the expression of CCL4 in murine macrophages. Mol Immunol 44: 1598-1605, 2007.

43. Herzig S, Long F, Jhala US, Hedrick S, Quinn R, Bauer A, Rudolph D, Schutz G, Yoon C, Puigserver P, et al: CREB regulates hepatic gluconeogenesis through the coactivator PGC-1. Nature 413: 179-183, 2001.

44. Lonardo A, Adinolfi LE, Loria P, Carulli N, Ruggiero G, et al: Steatosis and hepatitis $C$ virus: mechanisms and significance for hepatic and extrahepatic disease. Gastroenterology 126: 586-597, 2004.

45. Koike K: Hepatitis C as a metabolic disease: Implication for the pathogenesis of NASH. Hepatol Res 33: 145-150, 2005.

46. Kawaguchi Y and Mizuta T: Interaction between hepatitis $\mathrm{C}$ virus and metabolic factors. World J Gastroenterol 20: 2888-2901, 2014.

47. Syed GH, Amako Y and Siddiqui A: Hepatitis C virus hijacks host lipid metabolism. Trends Endocrinol Metab 21: 33-40, 2010.

48. Rynes J, Donohoe CD, Frommolt P, Brodesser S, Jindra M and Uhlirova M: Activating transcription factor 3 regulates immune and metabolic homeostasis. Mol Cell Biol 32: 3949-3962, 2012.

49. Kim J, Di Vizio D, Kim TK, Kim J, Kim M, Pelton K, Clinton SK, Hai T, Hwang D, Solomon KR, et al: The response of the prostate to circulating cholesterol: Activating transcription factor 3 (ATF3) as a prominent node in a cholesterol-sensing network. PLoS One 7: e39448, 2012. 